\title{
Market Conditions and General Practitioners' Referrals
}

\section{Tor Iversen \\ Institute of Health Management and Health Economics, Health Economics Research Programme, University of Oslo}

\section{Ching-to Albert MA}

Department of Economics, Boston University, and University of Oslo

\section{UNIVERSITY OF OSLO} HEALTH ECONOMICS RESEARCH PROGRAMME

Working paper 2009: 8

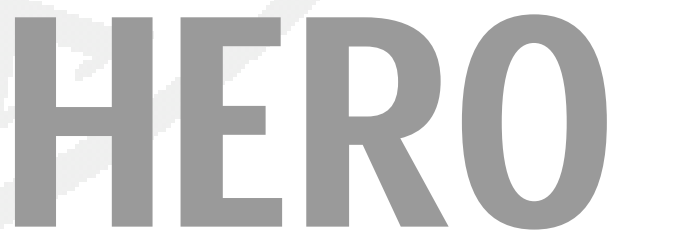




\title{
Market Conditions and General Practitioners' Referrals
}

\author{
Tor Iversen \\ Institute of Health Management and Health Economics, University of Oslo \\ P.O. Box 1089 Blindern, NO-0317 Oslo, Norway \\ E-mail: tor.iversen@medisin.uio.no \\ Ching-to Albert Ma \\ Department of Economics, Boston University, and University of Oslo \\ E-mail: $\underline{\text { ma@bu.edu }}$
}

September 2009

\section{Health Economics Research Programme at the University of Oslo HERO 2009}

Keywords:

JEL classification:

Physician, service motive, profit motive, referral, radiology

H42, I11, I18, C23

\section{Acknowledgement:}

We are grateful to the Research Council of Norway for financial support through the Health Economics Research Program at the University of Oslo (HERO). We thank seminar participants at Boston and Erasmus Universities, the University of York, the HEB/HERO Health Economics Workshop in Oslo 2009, Nordic Health Economists' Study Group in Reykjavik 2009 and especially Matthew Sutton for their comments and suggestions. The project is done in collaboration with The Norwegian Labour and Welfare Administration. The data file is prepared by Espen Halland Dahl and Jostein Ellingsen. Research assistance from Khalid Lafkiri is acknowledged. 


\begin{abstract}
We study how market conditions influence referrals of patients by general practitioners (GPs). We set up a model of GP referral for the Norwegian health care system, where a GP receives capitation payment based on the number of patients in his practice, as well as fee-for-service reimbursements. A GP may accept new patients or close the practice to new patients. We model GPs as partially altruistic, and compete for patients. We show that a GP operating in a more competitive market refers more. To retain patients in his practice, a GP satisfies patients' requests for referrals. Furthermore, a GP who faces patient shortage will refer more often than a GP who has enough patients. More referrals may add to profits from future treatments. Using data of radiology referrals by GPs in Norway, we test and confirm our theory.
\end{abstract}




\section{Introduction}

Nonprice rationing is common in health care delivery systems. Managed care, supply management, and gatekeeping are usual forms of rationing. In private health insurance markets in the United States, primary care physicians often coordinate care for patients. Specialty care is available only when a primary care physician refers the patient for such services. In many European countries, such gatekeeping is often the responsibility of a General Practitioner (GP). In this paper, we model a GP's referral decision, and assess its empirical significance.

We focus on GPs' radiology and imaging referrals. These services are diagnostic in nature, and yield valuable information to the GP. Often a radiology referral is the beginning of medical treatments by specialists. Radiology referrals by GPs are therefore key in cost control because specialty care uses more resource.

We study the factors that affect GPs' radiology referral decisions. Naturally, one expects that variations in patient characteristics would be correlated to the likelihood of referrals. Nevertheless, it is well known that these variations do not fully explain referrals. In this paper, we study how physician characteristics and local physician market conditions affect GPs' radiology referrals.

Our model is adapted for the Norwegian GP market. Since 2001, each inhabitant in Norway is listed with a GP. The size of a GP's practice is determined by a matching mechanism, and it is quite possible that a GP may want more patients than he currently has in the practice. A GP facing patient shortage may leave his practice open so that new patients may join. When the local physician market is competitive,

more GPs will have their practices open to new patients. We use the number of GPs with open practice in a municipality as a measure of the intensity of competition. Furthermore, we also use the information of whether a GP faces patient shortage to explain GPs' radiology referrals.

A GP is paid according to a capitation fee per patient in the practice and fee-for-service reimbursements. A GP does not directly benefit from referring a patient to radiology services. Our understanding is that Norwegian GPs have no financial stake in radiology laboratories. A GP may gain indirectly because he may provide more treatments to a patient after the radiology referral. Furthermore, radiology referrals may increase patient satisfaction. This may help the GP to retain patients and to attract new patients. 
A GP's preferences are a weighted sum of profits and patient benefit. These preferences are the key linkage between the theoretical model and the empirical implementation. We posit that these weights are influenced by market conditions. When the physician market is more competitive, the weight on patient benefit is assumed to be higher. A GP who has to compete against more rival GPs has a more liberal referral rule. The GP relaxes his gatekeeping rule to increase patient satisfaction level, preventing patients from leaving, and attracting new ones.

We consider as well the effect of patient shortage on referrals. A GP who faces patient shortage will have a higher referral rate than a GP who does not. This result is simply due to cost consideration. If GPs have increasing marginal costs, a GP with enough patients in his practice will reduce radiology referrals because the marginal cost of a referral is higher than if he had experienced a shortage of patients.

We test the predictions from the model with data on Norwegian GPs' referrals to diagnostic radiology. We have monthly radiology referral data at the individual physician level from 2004 to 2007. Our (unbalanced) panel consists of over 4,200 GPs, and over 165,000 referrals. The data set is unique since it includes information of all GPs in the Norwegian list patient system. We merge the referral data with data of physicians, so have information about physicians and their practices. Having practice size information, we can use a standardized dependent variable of referral rate per 1,000 patients.

Since each GP is likely to have his own practice style, observations of an individual GP are correlated over time. We estimate the contributions of potential explanatory variables by panel data methods, and control for unobserved heterogeneity by random effects and fixed effects.

First, we confirm that market conditions have the expected effects on radiology referrals. In a municipality with more open GP practices, a GP tends to refer more patients for radiology services. Second, we also confirm that a GP who faces a patient shortage refers more. Third, our empirical findings are consistent with common expectations: the referral rate declines with the distance between a GP and a radiology laboratory, GPs refer more when their patients are older, GPs with more female patients refer more, as do older GPs.

GP characteristics and market conditions contribute to the referral rate. The magnitude, though sta- 
tistically significant, does not seem to be large. Suppose we compare two municipalities, one with 0 open GP practice, and one about 50 open practices, which corresponds roughly to the 75 th percentile in ranking of municipalities with open practices. According to our estimates, the difference is 1.11 referrals per 1000 patients per month, just under a 9.5 percent increase from the mean referral rate.

An on-going health policy debate concerns the substitution of secondary care providers by primary care providers. This is usually regarded as a cost-saving strategy. By increasing the number of GPs, policy makers hope to reduce the use of more expensive specialists. The results here do not lend support to the cost-saving goal. When there are more GPs, competition becomes more intense, and increases radiology referrals, usually the gateway to specialty care. Our study is on radiology referral only, so it may not offer a comprehensive evaluation of the cost-saving argument.

Many papers in the health economics literature are on the relationship between financial incentives and such aspects of the health market as outcomes, costs, quantities and qualities. Physicians' responses against policy changes have been studied extensively (McGuire 2000, Leger 2008). Our paper here is on the relationship between gatekeeping and financial incentives, and the literature is smaller. Our study does not involve a policy implementation of new financial incentives. Rather, it follows a panel of physicians over a period of four years.

Gatekeeping by primary care physicians has been shown to be responsive to financial incentives. Dusheiko, Gravelle, Jacobs, and Smith (2006) study the United Kingdom fund holding natural experiment. Between 1991-1999, UK GPs who opted for a fund holding scheme were paid according to capitation, and responsible for their patients' elective surgery charges. These GPs have an incentive to avoid referring patients for such services. ${ }^{1}$ Dusheiko et al. show that the abolition of fund holding has increased elective surgery admission rates between $3.5 \%$ and $5.1 \%$.

Croxson, Propper, and Perkins (2001) find evidence that GPs in the UK do respond to financial incentives. They show that GPs have inflated cost for the time period before they become fundholders under capitation.

\footnotetext{
${ }^{1}$ In an experiment, Earwicker and Whynes (1998) show that GPs' referral rates are more responsive to specialist interests and waiting time than costs. Because the study consists of a questionaire survey, GPs do not actually have to bear any costs.
} 
Rochaix (1993) presents evidence for Quebec GPs responding to a price freeze during a fifteen month period by adjusting quantities. Generally, the evidence that physicians respond to financial incentives is widely accepted.

The financial incentives in Dusheiko et. al. and Croxson et. al. come directly from service capitation. In Rochaix (1993) GPs' reimbursement rates have been restrained. All three studies, as most in the literature, use a natural experiment, a policy change, to identify physicians' responses to changes in financial incentives. Any changes in market conditions then occur simultaneously with policy changes. By contrast, in our study, all GPs in the panel are subject to a common and time-invariant payment and capitation policy. The panel structure allows us to use exogenous market condition changes to identify GPs' responses.

The theoretical GP referral and gatekeeping literature in health economics has studied such issues as cream skimming (Barros and Olivella 2005), quality (Brekke, Nuscheler and Straume 2007), GP reputation and human capital investment (Gonzalez 2004), efficient use of information (Gonzalez 2009), and dual job incentives (Biglaiser and Ma 2007). The managed care literature (Glied 2000) has also considered the gatekeeping and referral role of GPs (Malcomson 2004), and diagnostic information and incentives (Allard, Jelovac, and Leger 2008). Except in Brekke et. al., these studies assume a fixed set of market conditions. In Brekke et. al. competition among specialists is studied in a model of referral under incomplete information. In our paper, market conditions vary exogenously, but we let GPs respond to market conditions by their referral decisions.

Our empirical implementation uses Norwegian GP data. Iversen and Lurås (2000) model a GP who decides on the number of patients in his practice and referrals of patients to specialists. A trial that began in 1993 for four municipalities replaced a GP's practice allowance by a capitation based on the practice size. Iversen and Lurås predict that this payment change would lead to a higher referral rate due to GPs shifting treatment costs to specialists. Norwegian data for one municipality before and after the introduction of capitation support the predictions.

Structured focus group interviews with Norwegian GPs by Carlsen and Norheim (2003) collaborate with the statistical results in Iversen and Lurås. The GPs generally perceived themselves as less concerned with 
the gatekeeper role under (partial) capitation scheme, and felt it more important to provide better services and keep patients satisfied. Lurås (2007) analyzes a survey of a representative sample of Norwegians on their satisfaction with their GPs. The satisfaction indexes include GP's interpersonal skills, medical skills, referral practices, and consultation lengths. Lurås finds that patients listed with GPs with patient shortage were less satisfied in all these dimensions than those listed with GPs with enough patients. Our paper complements these studies on the Norwegian health care system.

The rest of the paper is organized as follows. The next section describes parts of the Norwegian health care system relevant to our study. Section 3 presents a model of GP patient lists and referrals, as well as its predictions. Section 4 describes the data and summary statistics. The main regression results and robustness checks are in Section 5. The last section draws some conclusions.

\section{$2 \quad$ Study Setting}

Norway provides health care to its 4.5 million citizens by a national health service. Primary care physicians are mostly private practitioners, while hospitals are publicly owned with salaried physicians. The Regular General Practitioner Scheme implemented in 2001 requested that each inhabitant of Norway be listed with a General Practitioner (GP), a primary care physician. Over 95 percent of the population complied with the request. In a nonemergency episode of illness, a patient's initial medical services are provided by the GP. Secondary and specialty services require a GP's referral. Thus, besides providing medical services, the GP is a gatekeeper. ${ }^{2}$

About 90 percent of GPs are self-employed and contract with municipalities, and the remaining GPs are directly employed by municipalities. In 2004, a GP on average had between 1250 and 1300 patients listed in his or her practice. The establishment of a GP's patient list when the Regular General Practitioner Scheme started in 2001 is described as follows. First, each GP submits to the National Insurance Administration the maximum number of patients he is willing to take. Simultaneously, each inhabitant submits to the

\footnotetext{
${ }^{2}$ A patient may be able to obtain second opinions from a provider other than his or her own GP. In some cases, for example, when the patient is unable to get an appointment from the listed GP due to vacations or long waits, the patient can obtain services from another GP.
} 
Administration up to three preferred physicians. Second, a matching process respecting patients' and GPs' preferences form an initial patient list for a GP. Third, if a GP's maximum list size has been reached, his list is complete. If the initial match assigns less than a GP's maximum number of patients, the Administration then assigns to the GP inhabitants who have not submitted physician preferences. In 2001, 30 percent of the adult population did not submit any physician preferences. In June 2001, after this second round of assignments, about 30 percent of the GPs still had at least 100 patients less than their maximum number of patients. Some GPs may experience patient shortage.

In any year, a patient may switch GPs up to two times. A GP can announce whether his practice is open to new patients or not. A GP who already has the maximum number of patients may close his practice to new patients. The information on whether practices are open or closed to new patients is publicly available through the internet, or from the municipality.

In terms of out-of-pocket expenses, in 2006 a patient's copayments for an outpatient visit with a primary care physician and a specialist are respectively about US $\$ 25$ and US $\$ 40$. If within a year a patient's copayment exceeds US\$250, National Insurance Administration pays for the excess copayment. For a radiology consult, in addition to a copayment of US $\$ 30$, a patient will incur both time and transportation costs. In major cities, there may be many laboratories or hospitals where radiology and imaging facilities are available. In remote areas, however, such facilities may be less commonly available, and consumers may have to incur higher travel costs to fulfill a radiology referral.

A primary care physician earns three sources of income. First, a GP receives from the municipality a capitation payment for each patient listed with him. The capitation payment is not subject to risk adjustments. Second, a GP receives fee-for-service payments from National Insurance. Third, a GP receives patients' copayments. Each of the three components makes up about one third of a GP's practice income.

Radiology and imaging services in Norway are provided by public and private laboratories. Public laboratories are service departments of public hospitals. Private laboratories are for-profit companies. In either case, radiologists or trained medical personnel perform the procedures, and radiology reports are sent to the referring physicians. As far as we are aware, GPs in Norway do not have financial stakes or ownership 
in private radiology laboratories, although there is no legal restriction on partnership or joint ownership. Neither do we know of any contracts that specify payments from laboratories to GPs based on their radiology and imaging referrals.

Private laboratories charge patients copayments, and bill National Insurance for fees. With a referral a patient pays the same copayment whether he uses a public or private laboratory. A private laboratory must have a contract with a Regional Health Authority (RHA) to bill National Insurance Administration. The contract specifies the volume an RHA is prepared to pay for, and a payment amount. This revenue for a private laboratory is in addition to fees from National Insurance and patient copayment. If a laboratory provides services in excess of the contracted volume with an RHA, no extra payment will be received from the RHA, although the laboratory still receives reimbursement from National Insurance and patient copayment.

Many public and private radiology laboratories operate in major urban centers. The density of public laboratories in major cities is comparable to that of private laboratories. In rural areas, public hospitals are often the only facilities where imaging referrals can be served. A GP has the choice of referring a patient to a public laboratory or a private laboratory that has a contract with an RHA. In major cities private laboratories are often more convenient to patients because of shorter waiting and travel times, as well as

longer opening hours. Some public laboratories ration their services and do not accept ordinary referrals from primary care physicians. Nevertheless, for patients with more complicated or uncommon imaging and radiology procedures, laboratories in public hospitals may be more suitable. In any case, the typical referral seems more likely to be one for a private laboratory, and variations in market conditions for GPs will affect variations in referral rates to private labs.

\section{A Model of Referral and Patient List}

In this section we set up a model of radiology referral and patient list. The model will adopt many of the institutional features in the Norwegian general practitioner (GP) market. According to the National Insurance Administration, 99 percent of Norwegian residents are listed with a GP. The GP is also a gatekeeper; secondary services require a GP's referral. We focus on radiology and imaging referrals. We also model the 
GP's choice of optimal practice size.

We proceed in two steps. First, we analyze a GP's referral decision given the number of patients he has in the practice. Second, given the optimal referral decisions, we study the GP's choice of practice size. We focus on radiology referral. We normalize the demand for radiology services and assume that each patient in the GP's practice potentially requires a referral.

In an initial diagnostic visit, the GP obtains some information about a patient's expected benefit from a radiology referral. The GP also learns the cost the patient has to incur to follow through with the referral. The benefit information comes from the GP's assessment of the patient's medical condition, the likelihood that an imaging referral will yield useful information, and plans of further treatment after imaging results. The cost information refers to the patient's copayment, time and travel cost, as well as any relevant cost due to the referral.

The radiology referral may lead to further treatments, but negative imaging results may also indicate that no new services are needed. We do not model explicitly the continuing treatment process. Instead, we simply use a parameter $\beta$ to represent the expected benefit from a treatment episode beginning with a radiology referral. ${ }^{3}$ Let $\beta$ denote the net expected benefit from a radiology referral. Expected benefits vary across patients in the practice, so we let $\beta$ be random and follow the distribution $F$ with density $f$ on an interval, which will be defined shortly.

To the GP, a referral yields an expected net revenue $S$, and we allow this to depend on the patient's expected benefit $\beta$. The expected net revenue $S(\beta)$ summarizes potential patient copayments and fee-forservice reimbursements from National Insurance, and the GP's costs from the continuing treatment episode after the radiology referral. We do not have to impose stringent restrictions on the function $S$, but it likely follows an inverted U-shape. For low expected patient net benefits, the intensity of treatment may be low, and the GP expects to have a low net monetary return. For high expected patient net benefits, the GP may have to refer the patient to a specialist, and again, the net monetary return to the GP will be low. For medium values of expected patient net benefits, the GP will likely continue to provide treatments, and the

\footnotetext{
${ }^{3}$ This is sufficient for our purpose because we do not have data on patients' medical conditions at the time of the referral, or treatments after the referral decisions.
} 
net monetary return will be high.

Suppose that there are $n$ patients in the GP's practice. Each patient's expected net benefit from a referral $\beta$ is drawn independently from $F$. After an initial visit, the patient and the GP both learn the realization of $\beta$. Then the GP may suggest a referral and the patient will decide whether to follow through. We assume that the patient will accept a referral only if the net expected benefit is positive. We model the GP's referral decision by a net benefit threshold $\widehat{\beta}$ : he recommends a referral if and only if $\beta \geq \widehat{\beta}$. Because of the patient's reaction, we let $\widehat{\beta} \geq 0$. The total number of referrals is $n(1-F(\widehat{\beta}))$. There is therefore no loss of generality, if we let $\beta$ vary on the interval $[0,1]$; any negative realization of $\beta$ would not lead to a radiology consult.

The GP incurs monetary and time costs from referrals. These costs are due to the time and effort needed to read reports and communicate with radiologists, etc. We call this the effort cost due to referrals. Given that there are $n$ patients who demand referrals and the referral threshold $\widehat{\beta}$, the effort cost is $C(n(1-F(\widehat{\beta})))$, where $C$ is a positive, strictly increasing and strictly convex function.

The GP's expected payoff is

$$
n \int_{\widehat{\beta}}^{1} S(\beta) f(\beta) \mathrm{d} \beta-C(n(1-F(\widehat{\beta})))
$$

the first term is the monetary revenue, whereas the second term is the effort cost. We, however, model the physician's behavior as guided by the utility function

$$
U(\widehat{\beta} ; n, S, \theta) \equiv n \int_{\widehat{\beta}}^{1} S(\beta) f(\beta) \mathrm{d} \beta-C(n(1-F(\widehat{\beta})))+n \theta \int_{\widehat{\beta}}^{1} \beta f(\beta) \mathrm{d} \beta
$$

Besides the GP's revenue and effort, expression (1) includes a product of the patient's expected net surplus and a parameter $\theta$.

How do we interpret the utility function in (1)? We hypothesize that the GP's referral threshold is affected by market conditions. One can think of market conditions determining a bargaining outcome between physicians and patients. The bargaining outcome is given by the maximization of a weighted sum of the GP's payoff and the patient's surplus. Market conditions influence the weight on patient surplus. In a more competitive market, a GP will have less bargaining power because the patient may switch to another GP, and therefore the value of $\theta$ tends to be higher. In our empirical work, we use market conditions to 
identify $\theta$.

The GP's optimal choice of the referral threshold $\widehat{\beta}$ can be characterized as follows. The first-order derivative of (1) is

$$
\left[-S(\widehat{\beta})-\theta \widehat{\beta}+C^{\prime}(n(1-F(\widehat{\beta})))\right] f(\widehat{\beta}) n .
$$

At an interior solution, the value of $\widehat{\beta}$ is set where the weighted sum of the GP's and the patient's marginal benefits, $S(\widehat{\beta})+\theta \widehat{\beta}$, is equal to the marginal effort cost $C^{\prime}(n(1-F(\widehat{\beta})))$. At a corner solution, $\widehat{\beta}$ is set at 0 so that all patients with positive expected benefits will receive a referral.

How does the threshold $\widehat{\beta}$ change with respect to the number of patients in the practice $n$ and the market condition parameter $\theta$ ? Applying the implicit function theorem on the first-order condition (at the interior solution $),{ }^{4}$ we obtain the following:

- A GP decreases radiology referrals when the number of patient in his practice increases; $\partial \widehat{\beta} / \partial n>0$.

- A GP increases radiology referrals when the weight on patient benefit increases: $\partial \widehat{\beta} / \partial \theta<0$.

These results follow directly from benefit and cost considerations. A higher $\theta$ increases the concern for patient benefit, and induces the GP to lower the referral threshold, raising the number of referrals. When $n$ increases, the GP's marginal cost increases, which reduces referrals.

Next, we turn to the GP's decision on the optimal practice size. We begin by defining the optimized value of (1):

$$
V(n)=\max _{\widehat{\beta}} n \int_{\widehat{\beta}}^{1} S(\beta) f(\beta) \mathrm{d} \beta-C(n(1-F(\widehat{\beta})))+n \theta \int_{\widehat{\beta}}^{1} \beta f(\beta) \mathrm{d} \beta .
$$

The value function $V(n)$ is the GP's payoff from having $n$ patients in his practice when he chooses the optimal referral threshold. In the Norwegian system, the GP receives a capitation fee for each patient in the practice. Let $R$ denote the capitation fee. If the GP has $n$ patients, his total payoff is $V(n)+n R$, which we assume strictly quasi-concave.

\footnotetext{
${ }^{4}$ The sign of the derivative of the optimal referral threshold $\widehat{\beta}$ with respect to a parameter $(n$ and $\theta)$ is the same as the sign of the cross partial derivative of the objective function (1) with respect to $\widehat{\beta}$ and the parameter. Generally, if $x(\theta)=\operatorname{argmax}_{x} f(x ; \theta)$, then, by the implicit function theorem, $x^{\prime}(\theta)=-f_{x \theta} / f_{x x}$. and the sign of $x^{\prime}(\theta)$ is the same as the sign of $f_{x \theta}(x, \theta)$, by the second-order necessary condition for a maximum.
} 
If the GP were able to choose the practice size, he would choose it to maximize $V(n)+n R$. In the Norwegian system, however, the GP is only able to specify the desired practice size. Patients specify their preferences on GPs to National Health Insurance, and a central matching mechanism allocates patients to each GP. A GP may be assigned more or less than his desired number of patients. We assume that if a GP is assigned more than the desired number of patients for his practice, he rejects the excess number of patients. If he is assigned less than his desired number, then that becomes his practice size; we call this case patient shortage.

In the short run, a GP may not be able to influence the demand from patients who want to be listed with him. For a GP, we model patients' demand for listing with him as a random variable. Let $N$ be a random variable with support on the positive real number, and distribution $G$ and density $g$. The number of patients who want to be listed with a GP is a realization of $N$. Suppose that the GP chooses a desired practice size, $\widehat{N}$, which is the limit of his practice size. If $N<\widehat{N}$, the GP's practice size is $N$. If $N>\widehat{N}$, the GP's practice size is $\widehat{N}$.

Given the demand specification, for a given desired practice size $\widehat{N}$, the GP's expected payoff is

$$
\int_{0}^{\widehat{N}}[V(N)+N R] g(N) \mathrm{d} N+\int_{\widehat{N}}^{\infty}[V(\widehat{N})+\widehat{N} R] g(N) \mathrm{d} N .
$$

The first-order derivative of $(3)$ is

$$
\begin{aligned}
& {[V(\widehat{N})+\widehat{N} R] g(\widehat{N})-[V(\widehat{N})+\widehat{N} R] g(\widehat{N})+\left[V^{\prime}(\widehat{N})+R\right](1-G(\widehat{N})) } \\
= & {\left[V^{\prime}(\widehat{N})+R\right](1-G(\widehat{N})) . }
\end{aligned}
$$

The value of (4) vanishes if and only if $\widehat{N}=\operatorname{argmax}_{N} V(N)+N R \equiv N^{*}$. The optimal practice size limit is one that the GP would have chosen if he was able to pick the practice size freely.

The result captures the following intuition. Suppose that the GP sets a practice size limit $\widehat{N}$ below $N^{*}$. There are two possibilities. If $N$ turns out to be less than $\widehat{N}$, the payoff would not be affected by any small change in the practice limit. If $N$ is more than $\widehat{N}$, the GP would have increased his payoff by a small increase in $\widehat{N}$. Setting $\widehat{N}$ below $N^{*}$ is suboptimal. Now suppose that the GP sets a practice size limit $\widehat{N}$ above $N^{*}$. If $N$ is below $\widehat{N}$, the GP's payoff is unaffected by any small change in $\widehat{N}$. If $N$ is above $\widehat{N}$, the GP would 
have increased his payoff by a small decrease in $\widehat{N}$. It is indeed optimal for the GP to report truthfully his desired practice size. We summarize the result here:

- A GP optimally sets his desired practice size to the level he would have chosen if he were able to choose the practice size without any demand constraint; $\widehat{N}=N^{*} \equiv \operatorname{argmax}_{N} V(N)+N R$. The optimal practice size limit is independent of the distribution of demand $G$.

Market conditions may affect how likely a GP may face patient shortage. When the density of GPs in a geographical market is high, the GP may likely be rationed. According to the comparative static results above, he would recommend more radiology referral than a GP who does not face patient shortage. Furthermore, in a more competitive market, a GP may have less bargaining power, so that the value of $\theta$ tends to be higher. Both factors tend to raise a GP's equilibrium referral rates. We summarize the predictions of the model. Given a cost function $C$, distributions of referral benefits and demand, respectively $F$ and $G$ :

Prediction 1: A GP who faces patient shortage has a higher referral rate than a GP who does not.

Prediction 2: In a more competitive market, a GP is more likely to face patient shortage and to have less bargaining power, and therefore has a higher referral rate.

We now discuss the robustness of our model. We have focused on radiology referrals; our data are on such services. Including many services and referrals in the model would not involve any new conceptual issue. We can imagine that these other services or referral decisions are at their respective optimal levels. Then we can apply the comparative static results above. Furthermore, differences in patients' health conditions can be captured by altering the benefit distribution function $F$. Differences across municipalities and, hence, overall demand for GPs can be captured by altering patient distributions $G$.

We do not distinguish between referrals to public and those to private laboratories, but the model can be adapted for this distinction. Public laboratories are usually more appropriate for some medical conditions or when travel distances to private laboratories are too long. We can let the GP's referral decision be made in two steps. First, the GP decides how the patient may benefit from a diagnostic imaging test. In this first 
step, based on the patient's medical condition and location, the GP will have to consider whether a public laboratory or a private laboratory is more appropriate. Second, after the assessment of the benefit in the first step, he has to decide whether to make the referral, based on his utility (which is partially determined by the patient's benefit). For some medical conditions, public laboratories may be the only appropriate choice; this may also be true for some remote locations. Otherwise, the GP may have more flexibility in choosing between referrals to public and private laboratories. In our empirical analysis, we study separately referrals to private and public laboratories.

We have used a model with symmetric information. At the referral stage, the GP and the patient share the same information. The basic incentives in the model remain the same when there is asymmetric information. When the GP has more information, demand inducement becomes relevant. A patient may infer the benefit or cost of a referral from that recommendation. Nevertheless, along an equilibrium path, the patient's inference must be consistent with the GP's private information. Because our comparative results are based on simple utility maximization principles, we expect that the model predictions will continue to hold in a more complex environment with some asymmetric information.

We have assumed that a GP's demand is random. This is a natural assumption in the short run, say, within a year or two. In the long run, a GP likely has a higher demand if he has a good reputation, so we do expect that a GP will attempt to build reputation. By being more relaxed towards radiology referral, a GP may raise patient satisfaction, which enhances his reputation. Furthermore, in a more competitive environment, reputation should be more important. These considerations reinforce our results. We expect that in the long run, more referrals will be associated with a more competitive GP market.

\section{Descriptive statistics}

Data for this study are from the Norwegian National Insurance Administration. Claims data from private and public radiology laboratories are merged with information of referring physicians and characteristics of the physicians' municipalities. Only referrals from primary care physicians are included. Monthly numbers of referrals by a physician are available for the four years between 2004 and 2007. The referrals are classified 
according to four modalities: X-ray, ultrasound, magnetic resonance imaging (MRI), and computerized axial tomography (CT Scan). Because we are primarily interested in incentives of private GPs, we exclude salaried GPs employed by municipalities; this has led to a 7 percent reduction in the number of observations. Salaried GPs are mostly located in remote municipalities. Hence, even if we were interested in comparing private GPs with salaried GPs, it would be difficult to separate location and incentive effects.

Since we use claims data from the laboratories to the National Insurance, referrals that patients have refused are unavailable. Because we are interested in referrals due to competitive pressures and patients' demands, we do not consider this omission to be problematic. Although we have information about physicians and their practices, we do not have information about medical conditions of patients who have undergone the radiology referrals.

The population distribution in Norway is scattered. There are a number of fairly densely populated urban areas (such as Bergen, Oslo, and Trondheim), and many remote areas where long distances separate centers of municipalities. Patients referred to radiology examinations experience considerable variations of travel times depending on their locations. Since travel cost can be high, both in terms of transportation and time, GPs should be sensitive to patients' travel distances to their nearest radiology providers. We define the travel time with respect to a referral by the distance between the GP's municipality and the nearest laboratory's municipality. We use both travel times to private and public laboratories. Travel time is measured by the driving time in hours for a small private car. A matrix that describes the travel times between Norwegian municipalities in 2002 is provided by the private firm InfoMap Norge AS.

[Figure 1 about here.]

We define a GP's monthly referral rate as the ratio of the number of radiology referrals to the number of patients listed with him. We use a normalized referral rate per 1000 listed patients. Figure 1 shows GP's average monthly referral rates to public and private radiology laboratories. GPs' referrals to public and private laboratories relate differently to travel times. While GPs' referral rates to private radiology laboratories decline with travel times, the referral rates to public radiology in fact increase. The referral rates intersect at a travel time of about one and a half hours. The sum of private and public referral rates 
is approximately independent of the travel time.

[Table 1 about here.]

Table 1 reports the time trend of GPs' monthly referral rates to public and private laboratories. Both have increased over our data period. Data for 2004 are believed to be somewhat incomplete, especially for laboratories in public hospitals. Even disregarding 2004, we find a 13 percent increase in referrals to private radiology and a 17 percent increase in referrals to public radiology during the two years 2005-2007. There are some seasonal effects in our data. For example, GPs' referral rates in July are up to 60 percent lower than in November (this likely due to summer vacations). We will explain how we have handled the seasonal components at the end of Section 5 .

[Table 2 about here.]

Table 2 relates GPs' referral rates to the number of other GPs in the same municipality who accept new patients. The number of GPs with open lists is a measure of the degree of competition; patients dissatisfied with their current GPs can only switch to GPs who accept new patients. When the number of GPs with open list is large, we take it to mean that the GP market is more competitive. Relating to our model in Section 3, we use the number of GPs with open lists in a municipality as a proxy for the parameter $\theta$.

We have chosen to use administrative borders to define market boundaries. As an alternative, we could count the number of GPs with open lists who are within a certain distance from a GP, and use that as the extent of competition faced by the GP. This measure would be parallel to Propper, Burgess and Green (2004), who define a hospital's catchment area by a radius of thirty minutes of travel time, and measure the extent of competition by the number of hospitals in the catchment area. In Norway, primary care is the municipalities' responsibilities, and the overwhelming majority of the population are listed with GPs in their residential municipality. Therefore, administrative borders are appropriate for our study.

From Table 2, GPs' private laboratory referral rates are positively correlated with the number of GPs with open lists. Nevertheless, GPs' referral rates to public laboratories exhibit the opposite correlation. The 
total referral rate increases by 12.6 percent as the number of open lists moves from the median to above the 90 th percentile.

[Table 3 about here.]

Table 3 shows GPs' monthly referral rates according to whether or not they experience shortage of patients. A GP is said to experience a patient shortage if his actual list size is less than the maximum he has reported to National Insurance Administration by at least 100. In our data, an average of 24 percent of GPs have experienced patient shortage. The percentage of GPs with patient shortage is smaller in 2007 (21.9\%) than in 2004 (25.7\%). This reduction results from a drop in the reported maximum list sizes and an increase in the number of listed patients for some GPs. From Table 3, GPs who experience patient shortage refer more patients to private laboratories than those who have enough patients, but refer less patients to public laboratories. Nevertheless, the total referral rates are approximately equal between GPs who experience shortage and those who do not.

[Table 4 about here.]

Table 4 shows some characteristics of GP practices and their municipalities. Our data consist of a panel of GPs over a period of forty eight months, so in additional to the usual descriptives, we include between-GP and within-GP variations. ${ }^{5}$ The monthly total number of referrals is 19.0 per 1000 patients. From the columns that display between and within standard deviations we see that there are considerable variations both across individuals and between periods. The average list size is 1235 people. On average, 11 percent of the people listed are over 70 years old. Seventy percent of the GPs are male and the mean age of Norwegian GPs is 48 years. On average there are 74.5 practices that accept additional patients in the municipalities in the sample. This corresponds to a mean of 81 percent open lists. The mean travel time with car to the nearest private laboratory is 1 hour and 3 minutes. The longest travel time is more than 18 hours. The mean travel time to a public laboratory is 24 minutes with a maximum of 6 hours and 22 minutes. ${ }^{6}$

\footnotetext{
${ }^{5}$ Because our panel is unbalanced, the within and between standard deviations do not sum to the overall standard deviation.

${ }^{6}$ In Table 4, there are less observations for PrTravel and PuTravel than other variables. The reason is that InfoMap
} 
Only gender is a time-invariant variable, and the within variation equals to zero. Travel time comes out with some within variation due to 13 GPs having moved practices between municipalities during our data period. Finally, the patient composition variable shows variations over time.

\section{Empirical Analysis and Results}

We now use data described in Section 4 to test the predictions from the model in Section 3. For econometric analysis, the Norwegian list patient system has several advantages. There is almost complete patient and GP participation under Norwegian National Health Insurance. Self-selection into the system is unlikely to be a problem. We also know a GP's practice size. This allows us to standardize a GP's practice, so that comparing the number of referrals of GPs whose practices have different numbers of patients is meaningful. Physicians' fees from National Insurance and patient copayments are outcomes of negotiation between the state and the Norwegian Medical Association, and uniform among all GPs. At the municipality level, market conditions therefore do not influence a GP's fees.

Our data consist of radiology referrals made by a panel of doctors over a period of forty eight months. Unobserved heterogeneity among GPs is likely because our data cannot possibly include all relevant factors that influence referral decisions. Unobserved heterogeneity violates the assumptions of ordinary least squares regression because error terms of different periods may be correlated for a GP.

Using the index $i$ to refer to GP, and $t$ to refer to time period (a month), we fit a standard model:

$$
y_{i t}=\alpha_{i}+x_{i t} \beta+z_{i} \gamma+\epsilon_{i t} \quad(i=1, \ldots ., 4265 ; \quad t=1, \ldots ., 48) .
$$

The dependent variable $y_{i t}$ is the $i$-th GP's radiology referral rate in the $t$-th period. The independent variable $x_{i t}$ is a vector of GP and time varying explanatory variables, while $z_{i}$ is a vector of GP specific, time invariant variables. The vectors of coefficients to be estimated are $\beta$ and $\gamma$. To allow for GP heterogeneity, we let $\alpha_{i}+\epsilon_{i t}$ be a stochastic error term. The stochastic variable $\alpha_{i}$ is the GP specific random variable that

Norge AS provided the travel time information for 2002, but since then a number of municipalities merged, so the software could not provide information for new municipalities. 
captures unobserved heterogeneity. It differs among GPs, but is constant for a GP over time. We assume

$$
\begin{array}{lll}
\mathrm{E}\left(\epsilon_{i t}\right)=0 & \operatorname{Var}\left(\epsilon_{i t}\right)=\sigma_{\epsilon}^{2} & \operatorname{Cov}\left(\epsilon_{i t}, \epsilon_{i s}\right)=0 \\
\mathrm{E}\left(\alpha_{i}\right)=0 & \operatorname{Var}\left(\alpha_{i}\right)=\sigma_{\alpha}^{2} & \operatorname{Cov}\left(\alpha_{i}, \epsilon_{i t}\right)=0
\end{array}
$$

For the random-effects model to be valid, we need to have

$$
\operatorname{Cov}\left(\alpha_{i}, x_{i t}\right)=\operatorname{Cov}\left(\alpha_{i}, z_{i}\right)=0
$$

We test these two restrictions by a Hausman test. If the restriction is rejected, the fixed-effects model is selected. In the fixed-effects model $\alpha_{i}$ cancels, so the model is robust. When they are valid, randomeffects estimators are more efficient than the fixed-effects estimators. In addition, we can test for effects of time-invariant variables.

Our study focuses on market conditions. Two variables in our data set can serve to identify the extent of market competition, the parameter $\theta$ in Section 3. The first, \#Capacity, is the number of GPs in a municipality who accept new patients. ${ }^{7}$ The second, \% Capacity, is the proportion of GPs in a municipality who accept new patients. The variable \#Capacity correlates with the opportunity set available to a patient considering switching from his current GP. When \#Capacity is high, a patient should be able to switch between GPs more easily, and we interpret naturally that higher \#Capacity is more intense competition. Larger municipalities likely have larger values of \#Capacity. Nevertheless, longer travel distances between patients and GPs in larger municipalities may overstate the extent of competition. The variable \% Capacity is a sort of standardized measure of competition, so it may well control for some municipality size effect. In the analysis we use \#Capacity. We have also run the regressions with \% Capacity. The sign of the effect is not affected, but the null hypothesis in the Hausman test has been rejected more often. We argue that \#Capacity is predetermined because the number of GPs with open lists is arguably predetermined when referral decisions are being made. Geographical variations in the numbers of open lists are largely explained by variations in GP density prior to the introduction of the regular GP scheme in 2001. Referral decisions within a time period do not affect the number of open lists in the market within the same time period. Finally, our model yields predictions about referral rates depending on whether GPs face patient shortage,

\footnotetext{
${ }^{7}$ We define \#Capacity as the number of GPs in a municipality with open lists minus 1 if the GP has patient shortage; otherwise it is equal to the number of GPs with open lists.
} 
so we include the variable Short as defined in Table 4

[Table 5 about here.]

Results from the estimations of factors that influence a GP's referral rate to private radiology, PrReferral, are in Table 5. (In all tables, estimates that are significant at 5 percent are superscripted by ${ }^{*}$, and 1 percent by **.) Because the proportion of elderly in a GP's practice (Propold) and the GP's age (Age) show nonlinear relationships to PrReferral, we convert these two variables into dummy variables. For Propold groups are determined according to 10-th, 50-th and 95-th percentiles. We also have considered alternative groupings, but these have not changed regression results. Age is grouped according to the 75 percentile.

In Table 5 results are first presented for an Ordinary Least Squares regression, then a fixed-effects regression, and finally a random-effects regression. From the Hausman test, the null hypothesis of zero correlation between the explanatory variables and the individual stochastic term is not rejected at the conventional, 5 percent level. Hence, we describe the results from the random effects model.

Signs of estimates are as one would naturally expect. Referral rate increases with a patient's age and with the proportion of females in a GP's practice. Male GPs and older GPs refer more. GPs who experience a shortage of patients are estimated to have a referral rate 0.58 per 1000 listed patients greater than GPs with enough patients. Since the mean referral rate per 1000 listed patients is 11.8 per 1000 patients for GPs with enough patients, patient shortage contributes to a 4.9 percent increase in the referral rate. The estimated magnitude of the effect of \#Capacity is 0.021. Some impression of the magnitude of this effect is derived as follows. If we raise the number of GPs who accepts new patients from 0 to 53 , which is the 75 th percentile, we will add 1.11 to the referral rate, which is a 9.4 percent increase compared to the mean referral rate.

We have included an interaction term, \#Capacity*(1-Short), as an explanatory variable. The estimated coefficient assigned to the interaction term indicates the effect of capacity in the municipality for a GP with enough patients compared to a GP with patient shortage. Since competition is less of a threat for GPs with enough patients, we expect a negative estimate from this interaction term. From Table 5 this effect is indeed negative, but not statistically significant. 
[Table 6 about here.]

We check the robustness of the basic model by alternative estimation methods. First, GPs are clustered in municipalities, and GPs in the same municipality may develop their own professional culture of radiology referrals. This unobserved heterogeneity can be modelled in two ways. In the first specification, we introduce a dummy for each municipality. In the second specification, we introduce the municipality level as a third level with the random intercepts for GPs nested within the random intercepts for municipalities. Similar to the random intercept for the GP, we assume that the random intercept for a municipality has a zero expectation and a constant variance. In addition we assume that the random intercepts and residual error terms are mutually independent. From Table 6, neither signs nor magnitudes of the estimated coefficients of Short (0.46) nor \#Capacity (0.023) are changed much. We have found a statistically significant municipality random effect.

\section{[Table 7 about here.]}

Second, we divide our data according to the four radiology modalities, and estimate each individually. The results are in Table 7. We report estimates of the fixed-effects model for X-ray, ultrasound, and MRI, and the random-effects model for CT Scan; the Hausman test rejects random effects for all but CT Scan. We have dropped the interaction term because it was not statistically significant. From Table 7 the estimated coefficients of both Short and \#Capacity are positive and statistically significant at the one percent level. As expected, the magnitudes of the coefficients are smaller compared to the aggregate model where all modalities are put together. The absolute effects of Short are larger for X-ray and MRI. Evaluated at the mean referral rate of each modality the relative effect of short is about five percent for each modality, except for CT Scan which is seven percent. The relative effect of \#Capacity varies more according to modalities with ultrasound showing the largest relative effect and MRI the smallest.

[Table 8 about here.]

Finally, we let referrals to public laboratories and total referrals be dependent variables. These are normalized in the same way as referrals to private laboratories, at number of referrals in a month per 1000 
listed patients. The Hausman test rejects random effects for both regressions. From Table 8, Short has a positive effect on referrals to public laboratories. Similar to results in Table 5, Short has a positive effect on total referrals. The magnitude of the effect is about a five percent increase calculated from the mean. As expected, the distance to private laboratories has a positive effect on the referrals to public laboratories but no effect on the total number of referrals. The \#Capacity variable has a negative effect on referrals to public laboratories. This suggests that on average patients have better access to private than public laboratories. The effect of \#Capacity on the total number of referrals is positive.

As we have mentioned in Section 4, GPs' referral rates do exhibit some seasonal patterns. To explore the possible bias due to seasonality, we have run all the regressions with month dummies. The signs and magnitude of the effects of interest are hardly changed. Clearly, there is no need to remove seasonality completely by aggregating our data into annual observations. The month dummy implementations yield no consequences, and aggregation will cause us to lose too much information.

\section{Conclusions}

Gatekeeping is an integral part of managed care. General Practitioners, being primary care physicians, are frontline gatekeepers. In this paper, we examine how GPs' radiology referrals respond to market competition. When there are more available GPs in the market, a GP tends to refer more patients for radiology diagnostic tests. Using Norwegian data of GP radiology referrals, we show that the increase is statistically significant and modest. We have also shown that results are robust. Dividing our data into different modalities (X-ray, Ultrasound, MRI and CAT Scan), and allowing for municipality-location effects do not change our results.

Our results have important policy implications. Substituting secondary care by primary care is believed to be cost-saving by policy makers. The cost reduction due to this substitution seems quite obvious: primary care is less costly than specialty care. Nevertheless, increasing the number of GPs makes the primary physician market more competitive. Together with a capitation payment for a patient-list system, as in Norway, more GPs may experience patient shortage from the more intense competition. Our theoretical model shows that this may lead to more referrals, and our empirical results support that. Against more 
competition, GPs reoptimize by referring patients more often, responding positively to patient requests. The cost saving effect may be weakened by GPs' reactions. 


\section{References}

[1] Allard, Marie, Izabela Jelovac, and Pierre Thomas Leger, "Treatment and Referral Decisions under Different Physician Payment Mechanisms," 2008, HEC Montreal working paper.

[2] Pedro Pita Barros, and Pau Olivella, "Waiting Lists and Patient Selection," Journal of Economics 83 Management Strategy, Vol. 14, 2005, pp. 623-646.

[3] Biglaiser, Gary, and Ching-to Albert Ma, "Moonlighting: Public Service and Private Practice," Rand Journal of Economics, Vol. 38, 2007, pp. 1113-1133.

[4] Brekke, Kurt R., Robert Nuscheler, and Odd Rune Straume, "Gatekeeping in Health Care," Journal of Health Economics, Vol. 26, 2007, pp. 149-170.

[5] Carlsen, Benedicte, Norheim, Ole Frithjof, "Introduction of the Patient-list System in General Practice: Changes in Norwegian Physicians' Perception of Their Gatekeeper Role," Scandinavian Journal of Primary Health Care, Vol. 21, 2003, pp. 209-213.

[6] Croxson, Bronwyn, Carol Propper, and A. Perkins, "Do Doctors Respond to Financial Incentives? UK Family Doctors and the GP Fundholder Scheme," Journal of Public Economics, Vol. 79, 2001, pp.375-398.

[7] Dusheiko, Mark, Hugh Gravelle, Rowena Jacobs, and Peter Smith, "The Effect of Financial Incentives on Gatekeeping Doctors: Evidence from a Natural Experiment," Journal of Health Economics, Vol. 25, 2006, pp. 449-478.

[8] Earwicker, Stephen C., and David Whynes, "General Practitioners' Referral Thresholds and Choices of Referral Destination: an Experimental Study," Health Economics, Vol. 7, 1998, pp. 711-722.

[9] Glied, Sherry, "Managed Care," in Handbook of Health Economics, Anthony J. Culyer, and Joseph P. Newhouse, ed., Elsevier, Amsterdam, 2000.

[10] Gonzalez, Paula, "Should Physicians' Dual Practice be Limited? An Incentive Approach," Health Economics, Vol. 13, 2004, pp. 505-524. 
[11] Gonzalez, Paula, "The Gatekeeping Role of General Practitioners. Does Patients' Information Matter?" Health Economics, 2009, forthcoming.

[12] Iversen, Tor and Hilde Lurås, "The Effect of Capitation on GPs' Referral Decisions," Health Economics, Vol 9, 2000, pp. 199-210.

[13] Lurås, Hilde, "The Association between Patient Shortage and Patient Satisfaction with General Practitioners," Scandinavian Journal of Primary Health Care, Vol. 25, 2007, pp. 133-139.

[14] Leger, Pierre Thomas, "Physician Payment Mechanisms," in Financing Health Care: New Ideas for a Changing Society, Mingshan Lu and Egon Jonsson, ed., Wiley-Vch, Weinheim, pp. 149-176.

[15] McGuire, Thomas G., "Physician Agency," in Handbook of Health Economics, Anthony J. Culyer, and Joseph P. Newhouse, ed., Elsevier, Amsterdam, 2000.

[16] Malcomson, James, "Health Service Gatekeepers," Rand Journal of Economics, Vol. 35, No. 2, 2004, pp. 401-421.

[17] Propper, Carol, Simon Burgess, and Katherine Green, "Does competition between hospitals improve the quality of care? Hospital death rates and the NHS internal market," Journal of Public Economics, Vol. 88, 2004, pp. 1247-1272.

[18] Rochaix, Lise, "Financial Incentives for Physicians: The Quebec Experience," Health Economics, Vol. 2, 1993, pp. 163-176. 


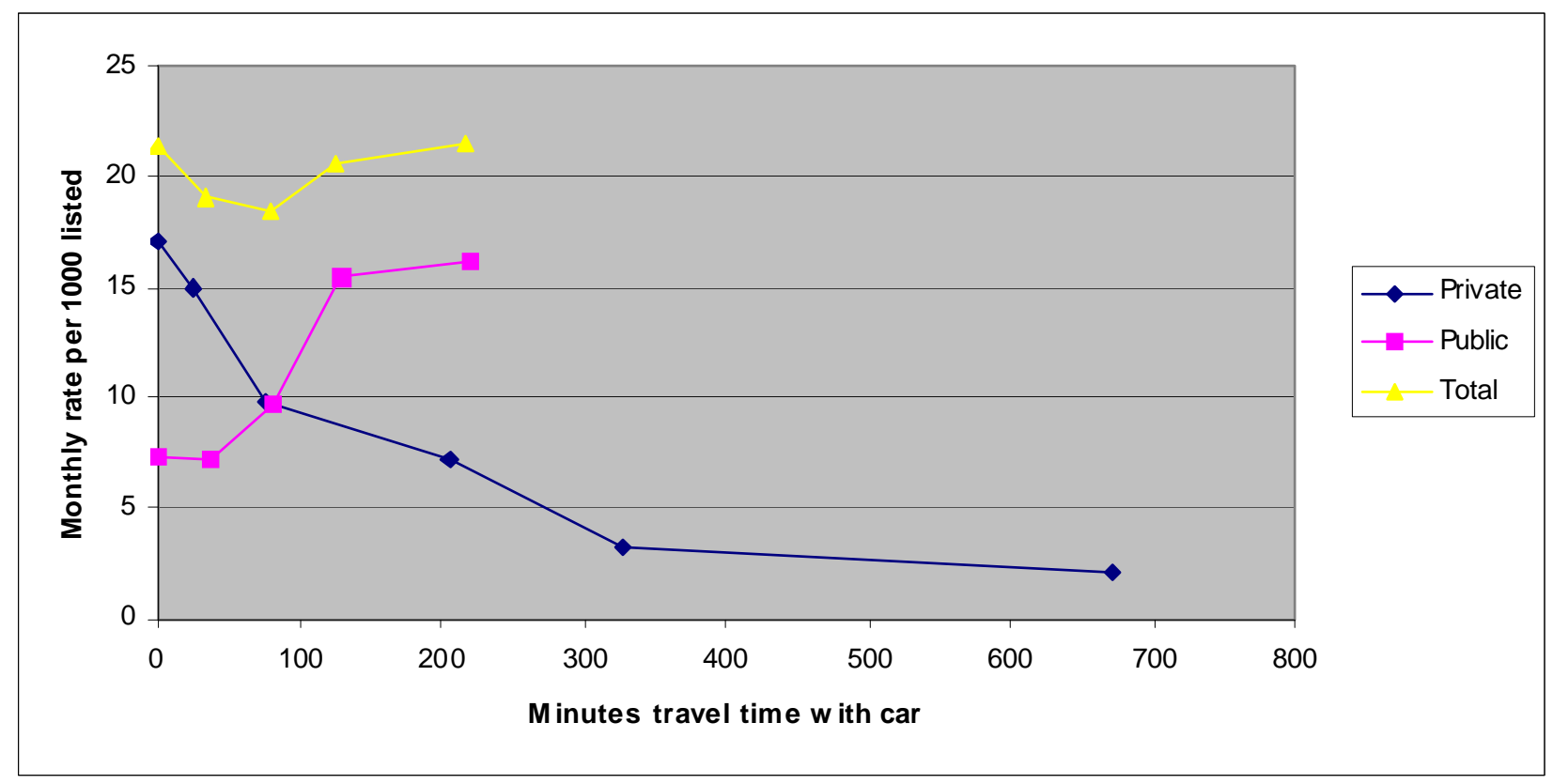

Figure 1: Travel time and referral rates

\begin{tabular}{|l|c|c|c|c|}
\hline Lab Type & & Year & & \\
\hline & $2004(39735)$ & $2005(41552)$ & $2006(41836)$ & $2007(42638)$ \\
\hline Private & 11.1 & 11.6 & 12.1 & 13.1 \\
\hline Public & 2.8 & 7.6 & 8.4 & 8.9 \\
\hline Total & 13.9 & 19.2 & 20.5 & 22.0 \\
\hline
\end{tabular}

Table 1: Monthly referral rates per 1000 listed patients, by years and laboratory types (\# obs. in parenthesis)

\begin{tabular}{|l|c|c|c|c|}
\hline & Median (165716) & 50th to 75 th\% (41249) & 75th to 90th\% (26372) & Over 90th\% (16970) \\
\hline Private & 12.0 & 12.3 & 15.0 & 20.6 \\
\hline Public & 7.0 & 7.3 & 3.1 & 0.8 \\
\hline Total & 19.0 & 19.6 & 18.1 & 21.4 \\
\hline
\end{tabular}

Table 2: Monthly referral rates per 1000 listed patients, by percentiles of open practices in municipality (\# obs. in parenthesis)

\begin{tabular}{|l|c|c|}
\hline & Enough patients (125922) & Shortage of patients (39839) \\
\hline Private & 11.8 & 12.6 \\
\hline Public & 7.2 & 6.3 \\
\hline Total & 19.0 & 18.8 \\
\hline
\end{tabular}

Table 3: Monthly referral rates per 1000 listed patients, by patient shortage (\# obs. in parenthesis) 


\begin{tabular}{|l|l|r|r|l|l|r|r|}
\hline Variable & Definition & mean & std.dev. & $\begin{array}{l}\text { std.dev. } \\
\text { between }\end{array}$ & $\begin{array}{l}\text { std.dev. } \\
\text { within }\end{array}$ & min & max \\
\hline Referral & $\begin{array}{l}\text { No. referrals in month per 1000 } \\
\text { listed patients }\end{array}$ & 19.0 & 12.5 & 9.1 & 9.1 & 0 & 500 \\
\hline PrReferral & $\begin{array}{l}\text { No. referrals in month per 1000 } \\
\text { listed patients to private provider }\end{array}$ & 12.0 & 10.1 & 8.4 & 6.1 & 0 & 500 \\
\hline PuReferral & $\begin{array}{l}\text { No. referrals in month per 1000 } \\
\text { listed patients to public provider }\end{array}$ & 7.0 & 9.9 & 7.8 & 6.4 & 0 & 249 \\
\hline List & $\begin{array}{l}\text { Practice size in units of 1000 pa- } \\
\text { tients }\end{array}$ & 1.235 & 0.390 & 0.399 & 0.081 & 0.09 & 2.712 \\
\hline Propold & $\begin{array}{l}\text { Proportion of persons in the list } \\
\text { aged 70 and older }\end{array}$ & 0.11 & 0.06 & 0.06 & 0.009 & 0.001 & 0.68 \\
\hline Propfem & Proportion of females in the list & 0.51 & 0.10 & 0.10 & 0.012 & 0.09 & 0.90 \\
\hline Male & $\begin{array}{l}\text { Dummy variable equal to one if } \\
\text { the physician is male }\end{array}$ & 0.70 & 0.46 & 0.46 & 0 & 0 & 1 \\
\hline Age & GP's age in years in 2001 & 48.0 & 9.41 & 10.2 & 1.13 & 26 & 75 \\
\hline Short & $\begin{array}{l}\text { Proportion of GPs with (pre- } \\
\text { ferred - actual list) } 100\end{array}$ & 0.24 & 0.43 & 0.40 & 0.21 & 0 & 1 \\
\hline \#Capacity & $\begin{array}{l}\text { No. GPs in municipality who ac- } \\
\text { cept new patients }\end{array}$ & 74.5 & 129.4 & 124.5 & 7.8 & 0 & 422 \\
\hline \%Capacity & $\begin{array}{l}\text { Proportion GPs in municipality } \\
\text { who accept new patients }\end{array}$ & 0.81 & 0.15 & 0.12 & 0.09 & 0 & 1 \\
\hline PrTravel & $\begin{array}{l}\text { Travel time in hours by car from } \\
\text { GP's municipality to nearest pri- } \\
\text { vate laboratory's municipality }\end{array}$ & 1.04 & 2.00 & 2.21 & 0.17 & 0 & 18.42 \\
\hline PuTravel & $\begin{array}{l}\text { Travel time in hours by car from } \\
\text { GP's municipality to nearest pub- } \\
\text { lic laboratory's municipality }\end{array}$ & 0.40 & 0.71 & 0.82 & 0.09 & 0 & 6.37 \\
\hline
\end{tabular}

Table 4: Variable definitions and deacriptives. There are 165425 observations for each variable, except the last two. There are 165283 observations for each of PrTravel and PuTravel. 


\begin{tabular}{|l|l|l|l|}
\hline & \multicolumn{3}{|c|}{ PrReferral } \\
\hline & OLS & Fixed effects & Random effects \\
\hline Propfem & $5.07^{* *}(0.33)$ & $9.56^{* *}(1.24)$ & $7.82^{* *}(0.96)$ \\
\hline $0.042<$ Propold $\leq 0.105$ & $0.80^{* *}(0.08)$ & $0.77^{* *}(0.14)$ & $0.80^{* *}(0.13)$ \\
\hline $0.105<$ Propold $\leq 0.217$ & $1.35^{* *}(0.08)$ & $1.00^{* *}(0.17)$ & $1.07^{* *}(0.16)$ \\
\hline $0.217<$ Propold & $2.21^{* *}(0.13)$ & $1.96^{* *}(0.26)$ & $2.01^{* *}(0.24)$ \\
\hline Male & $1.20^{* *}(0.07)$ & & $1.57^{* *}(0.27)$ \\
\hline $55 \leq$ Age & $0.65^{* *}(0.05)$ & $0.092(0.097)$ & $0.16^{* *}(0.09)$ \\
\hline Short & $0.52^{* *}(0.06)$ & $0.57^{* *}(0.08)$ & $0.58^{* *}(0.08)$ \\
\hline \#Capacity & $0.022^{* *}(0.0003)$ & $0.016^{* *}(0.0002)$ & $0.021^{* *}(0.00008)$ \\
\hline \#Capacity*(1-Short) & $0.003^{* *}(0.0004)$ & $-0.0009(0.0005)$ & $-0.0005(0.0005)$ \\
\hline PrTravel & $-1.58^{* *}(0.01)$ & $-1.57^{* *}(0.10)$ & $-1.52^{* *}(0.05)$ \\
\hline PuTravel & $0.58^{* *}(0.04)$ & $0.58^{*}(0.20)$ & $0.54^{* *}(0.13)$ \\
\hline Constant & $8.23^{* *}(0.2)$ & $7.59^{* *}(0.67)$ & $6.84^{* *}(0.62)$ \\
\hline Dummies for years & Yes & Yes & Yes \\
\hline Dummies for municipalities & No & No & No \\
\hline Adjusted R ${ }^{2}$ & 0.21 & 0.20 & 0.21 \\
\hline No.observations & 165092 & 165092 & 165092 \\
\hline No.GPs & & 4261 & 4261 \\
\hline No.observations per GP & & Min:1;Avg:38.7; Max:48 & Min:1;Avg:38.7; Max:48 \\
\hline$\hat{\sigma}_{\alpha}^{2}$ & & & 0.57 \\
\hline$\hat{\sigma}_{\alpha}^{2}+\hat{\sigma}_{\epsilon}^{2}$ & & & $\chi^{2}(12)=20.51 ; \mathrm{p}=0.058$ \\
\hline Hausman test & & & \\
\hline
\end{tabular}

Table 5: Estimated effect (robust std) of practice characteristics and market conditions on the monthly number of referrals to private laboratories per 1000 listed patients

\begin{tabular}{|l|l|l|}
\hline & \multicolumn{2}{|c|}{ PrReferral } \\
\hline & Municipalities as fixed effects & Municipalities as random intercept \\
\hline Propfem & $9.44^{* *}(1.38)$ & $6.79^{* *}(0.88)$ \\
\hline $0.042<$ Propold $\leq 0.105$ & $0.90^{* *}(0.14)$ & $0.89^{* *}(0.13)$ \\
\hline $0.105<$ Propold $\leq 0.217$ & $1.30^{* *}(0.18)$ & $1.57^{* *}(0.16)$ \\
\hline $0.217<$ Propold & $2.08^{* *}(0.27)$ & $2.51^{* *}(0.23)$ \\
\hline Male & & $1.39^{* *}(0.21)$ \\
\hline $55 \leq$ Age & $0.11(0.10)$ & $0.24^{* *}(0.09)$ \\
\hline Short & $0.46^{* *}(0.08)$ & $0.45^{* *}(0.08)$ \\
\hline \#Capacity & $0.023^{* *}(0.004)$ & $0.025^{* *}(0.003)$ \\
\hline \#Capacity*(1-Short) & $-0.0012^{*}(0.0005)$ & $-0.0006(0.0005)$ \\
\hline PrTravel & $-1.71(1.65)$ & $-1.15^{* *}(0.11)$ \\
\hline PuTravel & $0.21(4.82)$ & $0.31(0.31)$ \\
\hline Constant & $4.53^{* *}(1.30)$ & $5.95^{* *}(0.67)$ \\
\hline Dummies for years & Yes & Yes \\
\hline Dummies for municipalities & Yes & No, random intercept \\
\hline Adjusted R ${ }^{2}$ & 0.15 & \\
\hline No.observations & 165092 & 165092 \\
\hline No.GPs & 4261 & 4261 \\
\hline No.observations per GP & Min:1;Avg:38.7; Max:48 & Min:1;Avg:38.7; Max:48 \\
\hline Var at municipality level & & $4.64(0.20)$ \\
\hline Hausman test & $\chi^{2}(136)=691.08 ;$ p $=0.000$ & \\
\hline
\end{tabular}

Table 6: Estimated effect (robust std) of practice characteristics and market conditions on the monthly number of referrals to private laboratories per 1000 listed patients adjusted for the municipality level 


\begin{tabular}{|l|l|l|l|l|}
\hline & \multicolumn{5}{|c|}{ PrReferral } \\
\hline & $\begin{array}{l}\text { X-ray } \\
\text { (fixed effects) }\end{array}$ & $\begin{array}{l}\text { Ultrasound } \\
\text { (fixed effects) }\end{array}$ & $\begin{array}{l}\text { MRI } \\
\text { (fixed effects })\end{array}$ & $\begin{array}{l}\text { CT Scan } \\
\text { (random effects })\end{array}$ \\
\hline Short & $0.28^{* *}(0.04)$ & $0.07^{* *}(0.015)$ & $0.19^{* *}(0.03)$ & $0.10^{* *}(0.02)$ \\
\hline \#Capacity & $0.011^{* *}(0.001)$ & $0.043^{* *}(0.004)$ & $0.001^{* *}(0.0008)$ & $0.004^{* *}(0.0001)$ \\
\hline PrTravel & $-0.74^{* *}(0.05)$ & $-0.16^{* *}(0.023)$ & $-0.47^{* *}(0.04)$ & $-0.20^{* *}(0.009)$ \\
\hline PuTravel & $0.37^{* *}(0.11)$ & $0.03(0.05)$ & $0.16(0.09)$ & $0.04(0.03)$ \\
\hline Year dummy & Yes & Yes & Yes & Yes \\
\hline Municipality dummy & No & No & No & \\
\hline Adjusted R ${ }^{2}$ & 0.19 & 0.12 & 0.07 & 0.13 \\
\hline Observations & 165092 & 165092 & 165092 & 165092 \\
\hline GPs & 4261 & 4261 & 4261 & 4261 \\
\hline Observations/GP & Min:1;Avg:38.7; & Min:1;Avg:38.7; & Min:1;Avg:38.7; & $\begin{array}{l}\text { Min:1;Avg:38.7; } \\
\text { Max:48 }\end{array}$ \\
\hline$\hat{\sigma}_{\alpha}^{2}$ & Max:48 & Max:48 & & 0.37 \\
\hline$\hat{\sigma}_{\alpha}^{2}+\hat{\sigma}_{\epsilon}^{2}$ & & & & $\chi^{2}(12)=16.63 ;$ \\
& Hausman test & $\chi^{2}(12)=25.80 ;$ & $\chi^{2}(12)=28.71 ;$ & $\chi^{2}(12)=41.54 ;$ \\
$\mathrm{p}=0.011$ & $\mathrm{p}=0.004$ & 0.000 & $\mathrm{p}=0.16$ \\
\hline
\end{tabular}

Table 7: Estimated effect (robust std) of practice characteristics and market conditions on the monthly number of referrals to private laboratories according to modalities per 1000 listed patients

\begin{tabular}{|l|l|l|}
\hline & \multicolumn{1}{|c|}{ PuReferral } & Referral \\
\hline & Fixed effects & Fixed effects \\
\hline Propfem & $2.96^{*}(1.28)$ & $12.52^{* *}(1.80)$ \\
\hline $0.042<$ Propold $\leq 0.105$ & $0.73^{* *}(0.14)$ & $1.5^{* *}(0.20)$ \\
\hline $0.105<$ Propold $\leq 0.217$ & $1.31^{* *}(0.18)$ & $2.29^{* *}(0.25)$ \\
\hline $0.217<$ Propold & $2.21^{* *}(0.27)$ & $4.17^{* *}(0.38)$ \\
\hline Male & & \\
\hline $55 \leq$ Age & $0.62^{* *}(0.10)$ & $0.77^{* *}(0.14)$ \\
\hline Short & $0.17^{*}(0.08)$ & $0.73^{* *}(0.12)$ \\
\hline \#Capacity & $-0.011^{* *}(0.002)$ & $0.0054^{*}(0.002)$ \\
\hline \#Capacity*(1-Short $)$ & $-0.003^{* *}(0.0005)$ & $-0.0043^{* *}(0.0007)$ \\
\hline PrTravel & $1.64^{* *}(0.11)$ & $0.08(0.15)$ \\
\hline PuTravel & $-0.91^{* *}(0.21)$ & $-0.33(0.30)$ \\
\hline Constant & $5.69^{* *}(0.69)$ & $13.28^{* *}(0.97)$ \\
\hline Dummies for years & Yes & Yes \\
\hline Adjusted R ${ }^{2}$ & 0.22 & 0.07 \\
\hline No. observations & 165092 & 165092 \\
\hline No. GPs & 4261 & 4261 \\
\hline No. observations per GP & Min:1;Avg:38.7; Max:48 & Min:1;Avg:38.7; Max:48 \\
\hline Hausman test & $\chi^{2}(13)=129.42 ; \mathrm{p}=0.000$ & $\chi^{2}(13)=116.85 ; \mathrm{p}=0.000$ \\
\hline
\end{tabular}

Table 8: Estimated effect (robust std) of practice characteristics and market conditions on the monthly number of referrals to public laboratories and total number of referrals per 1000 listed patients 